\title{
Deep near-IR variability survey of pre-main-sequence stars in $\rho$ Ophiuchi ${ }^{\star}$
}

\author{
C. Alves de Oliveira and M. Casali
}

\author{
European Southern Observatory (ESO), Karl-Schwarzschild-Strasse 2, 85748 Garching bei Muenchen, Germany \\ e-mail: coliveir@eso.org
}

Received 26 November 2007 / Accepted 1 April 2008

\begin{abstract}
Context. Variability is a common characteristic of pre-main-sequence stars (PMS). Near-IR variability surveys of young stellar objects (YSOs) can probe stellar and circumstellar environments and provide information about the dynamics of the ongoing magnetic and accretion processes. Furthermore, variability can be used as a tool to uncover new cluster members in star formation regions.

Aims. We hope to achieve the deepest near-IR variability study of YSOs targeting the $\rho$ Ophiuchi cluster.

Methods. Fourteen epochs of observations were obtained with the Wide Field Camera (WFCAM) at the UKIRT telescope scheduled in a manner that allowed the study of variability on timescales of days, months, and years. Statistical tools, such as the multi-band cross correlation index and the reduced chi-square, were used to disentangle signals of variability from noise. Variability characteristics are compared to existing models of YSOs in order to relate them to physical processes, and then used to select new candidate members of this star-forming region.

Results. Variability in the near-IR is found to be present in $41 \%$ of the known population of $\rho$ Ophiuchi recovered in our sample. The behaviours shown are several and can be associated with the existence of spots on the stellar surface, variations in circumstellar extinction, or changes in the geometry of an accretion disc. Using variability, a new population of objects has been uncovered that is believed to be part of the $\rho$ Ophiuchi cluster.
\end{abstract}

Key words. stars: pre-main-sequence - stars: low-mass, brown dwarfs - stars: activity - stars: variables: general

\section{Introduction}

Young stars have been known to be variable since Joy (1945) described the irregular behaviour of T Tauri stars. Photometric variability is thought to originate from several mechanisms related to magnetic fields, accretion discs, and circumstellar extinction (Herbst et al. 1994). Optical variability has been used as an excellent tool for characterising stellar and circumstellar environments of pre-main-sequence stars (e.g., Grankin et al. 2007, 2008) and brown dwarfs (e.g., Caballero et al. 2004; Scholz \& Eislöffel 2005). The mechanisms causing optical variability in YSOs are also thought to be responsible for the occurrence of photometric variations in the near-IR, especially suitable for probing phenomena which take place in the circumstellar temperature regime (see Eiroa et al. 2002, and references therein). Furthermore, Kaas (1999) has shown that IR variability is a very useful tool for sorting the cluster population from the background field. For instance, it has the advantage of identifying young sources that do not show IR-excess or have small $\mathrm{H} \alpha$ emission, as is the case for weak-line T Tauri stars (WTTS), but are magnetically active (Grankin et al. 2008). The use of this relatively new technique should contribute significantly to obtain a full census of the young objects in star formation regions, one of the most important goals of galactic star formation studies.

At a distance of $119 \pm 6$ pc (Lombardi et al. 2008), the $\rho$ Ophiuchi cloud core is one of the nearest star forming regions, and therefore widely studied. Despite the proximity, few

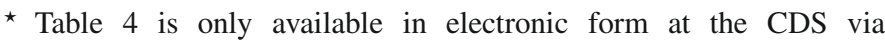
anonymous ftp to cdsarc.u-strasbg.fr (130.79.128.5) or via http://cdsweb.u-strasbg.fr/cgi-bin/qcat?J/A+A/485/155 stars are optically observable due to the high visual extinction in the core, estimated to be 50-100 mag (Wilking \& Lada 1983). That is also the case for most Myr old clusters, where much of the interesting population remains visibly obscured, and must be studied with IR observations. However, with many nearby star forming regions extending over degree scales and IR cameras usually having fields of only arc minutes, obtaining a single image of a region such as $\rho$ Ophiuchi has been a major project until now, let alone attempting multi-epoch studies. This has changed with 2MASS which allows large area, though rather shallow, observations to be made, and near-IR variability studies to become possible (Carpenter et al. 2001, 2002). In a 2MASS JHK study centred near the Trapezium region of the Orion Nebula Cluster, where most star formation is thought to have occurred between 0.3 and 2 Myr ago (Ali \& Depoy 1995; Hillenbrand 1997), Carpenter et al. (2001) found that approximately $45 \%$ of young stars were variable.

More recently, the WFCAM IR imager (Casali et al. 2007) has become operational on the UK Infrared Telescope (UKIRT). The large field of view ( $\sim 0.8 \mathrm{deg}^{2}$ in four exposures) of this instrument has made deep variability studies possible for the first time in the IR. This paper presents the results of such a study, with multi-epoch $H$ and $K$ observations of the $\rho$ Ophiuchi cluster used to search for variability on timescales of days, months, and years. The study is enhanced by including recently released IRAC/Spitzer data on $\rho$ Ophiuchi (Evans et al. 2005). In Sect. 2, the observations and reductions for the large data sample are described. Section 3 explains the methods used in the search for variability together with the results of the analysis. These are discussed and explained in a physical context in Sect. 4 , in an 
Table 1. Coordinates of the observations.

\begin{tabular}{ccc}
\hline \hline Pointing & RA & Dec \\
\hline 1 & 162720.0 & -241920 \\
2 & 162720.0 & -243234 \\
3 & 162818.2 & -242900 \\
4 & 162818.2 & -241548 \\
\hline
\end{tabular}

Table 2. Log of the observations.

\begin{tabular}{lccc}
\hline \hline UT date & May & June & July \\
2005 & $11,18,21,29$ & $04,07,14$ & $\ldots$ \\
\hline 2006 & $24,28^{a}$ & $03,14,23$ & 04,10 \\
\hline
\end{tabular}

attempt to relate the variability behaviours observed to the possible physical causes. Conclusions and future prospects are given in Sect. 5.

\section{Observations}

The WFCAM on the UKIRT is a survey instrument developed at the UK Astronomical Technology Centre (UK ATC), primarily for the UKIRT Infrared Deep Sky Survey (UKIDSS) (Lawrence et al. 2007). WFCAM is a wide field imaging camera operating in the near infrared from $0.83 \mu \mathrm{m}$ to $2.37 \mu \mathrm{m}$ in up to eight filters, including $Z Y J H K$ (Hewett et al. 2006). The camera uses four Rockwell Hawaii-II $2048 \times 204818 \mu$ m-pixel array detectors with a pixel scale of $0.4^{\prime \prime}$. The four detectors are arranged in a $2 \times 2$ pattern and are separated by $94 \%$ of a detector width, for which four exposures are needed to survey a contiguous area of $\sim 0.8 \mathrm{deg}^{2}$ (Casali et al. 2007).

The data presented amount to a total of 14 epochs in the $\rho$ Ophiuchi cluster. The observed region consists of four exposures which produce a final tile of $\sim 0.8 \mathrm{deg}^{2}$ centred on the cloud's core. Each observing block was done in $H$ and $K$ bands where completeness limits of magnitudes 19.0 and 18.0, respectively, are achieved. Table 1 shows the central position (right ascension and declination) for each of the four exposures. The same region in the sky was observed randomly over scales of days, weeks, months, and year sampling periods. Table 2 provides a list of the nights in which the observations occurred.

To complement the study, Spitzer data from the c2d legacy project (Evans et al. 2003) have been included. The Ophiuchus molecular cloud has been mapped with IRAC (Fazio et al. 2004) in the 3.6, 4.5, 5.8 and $8.0 \mu \mathrm{m}$ bands, over a region of $8.0 \mathrm{deg}^{2}$, which encompasses the WFCAM field. The data have been retrieved from the preliminary c2d IRAC point-source catalogues of the third data delivery (Evans et al. 2005).

\subsection{Data processing}

WFCAM data are processed and archived by the VISTA Data Flow System Project, a collaboration between Queen Mary University of London, the Institute of Astronomy of the University of Cambridge and the Institute for Astronomy of the University of Edinburgh (Irwin et al., in preparation). The pipeline flat-fields the data, subtracts the counts from the background sky, detects and parameterises objects, and performs the photometric and astrometric calibrations. The products from the pipeline are a set of reduced uncalibrated individual exposures, and photometrically and astrometrically
Table 3. Photometric accuracy.

\begin{tabular}{ccc}
\hline \hline Magnitude & \multicolumn{2}{c}{ Photometric Accuracy } \\
& $H$ & $K$ \\
\hline $10-11$ & $\ldots$ & 0.01 \\
$11-12$ & 0.01 & 0.01 \\
$12-13$ & 0.01 & 0.01 \\
$13-14$ & 0.01 & 0.01 \\
$14-15$ & 0.01 & 0.01 \\
$15-16$ & 0.01 & 0.02 \\
$16-17$ & 0.02 & 0.05 \\
$17-18$ & 0.04 & 0.07 \\
$18-19$ & 0.05 & $\ldots$ \\
\hline
\end{tabular}

calibrated stacked frames, as well as catalogues of sources detected in the frames and ingested into the WFCAM Science Archive, described by Hambly et al. (2008). A summary description of the WFCAM pipeline and science archive is given by Lawrence et al. (2007).

Given the expected very low amplitudes of variation in young stars, a few tenths or less of a magnitude in the near-IR (Carpenter et al. 2001), it is important to be aware of common problems intrinsic to the instrument and type of observations. To a large extent, many of these problems are eliminated by the pipeline during reduction and calibration. However, others do persist giving rise to false detections which show up in the catalogues. Although they represent a small fraction of the dataset, they need to be filtered out. A routine was written in IDL to implement an algorithm which finds and removes these spurious sources and implements the necessary constraints to ensure a reliable final source list. These are briefly explained below.

- Overlapping regions: the algorithm accounted for sources in the overlapping regions between detectors which have multiple entries in the catalogue, keeping the source with the largest distance to the detector edge.

- Magnitude limits: sets a cut-off limit at the brighter end (magnitudes 11.0 and 10.0 for $H$ and $K$, respectively) due to saturation. At the fainter end, magnitudes 19.0 and 18.0 for $H$ and $K$, respectively, mark the completeness limits which are above the $10 \sigma$ level (see Table 3 for the average errors).

- Object classification: objects flagged by the pipeline as stars were kept as well as objects which were classified as galaxies but showed no difference in magnitude between different apertures. The motivation was to keep some nebulous YSOs while setting the criteria strictly enough to avoid galaxy contamination.

- Cross-talk: electronic cross-talk occurs between the detector channels within a quadrant. It produces a sequence of spurious images in some or all the other 7 channels (Dye et al. 2006). The algorithm removed sources located at up to seven multiples of 128 pix from bright stars.

- Diffraction patterns: sources in the surroundings of saturated stars were removed since their photometry is not reliable. The radius of rejection was adjusted according to the brightness of the star.

- Field edges: sources located on the outer field edge were removed since they were found to often have unreliable photometry.

Furthermore, the $K$ band images from the observations made on 28 May 2006 were discarded since they were of lower quality due to technical problems.

The catalogues for each night were merged for the two years separately. Sources were matched by requiring their position to 


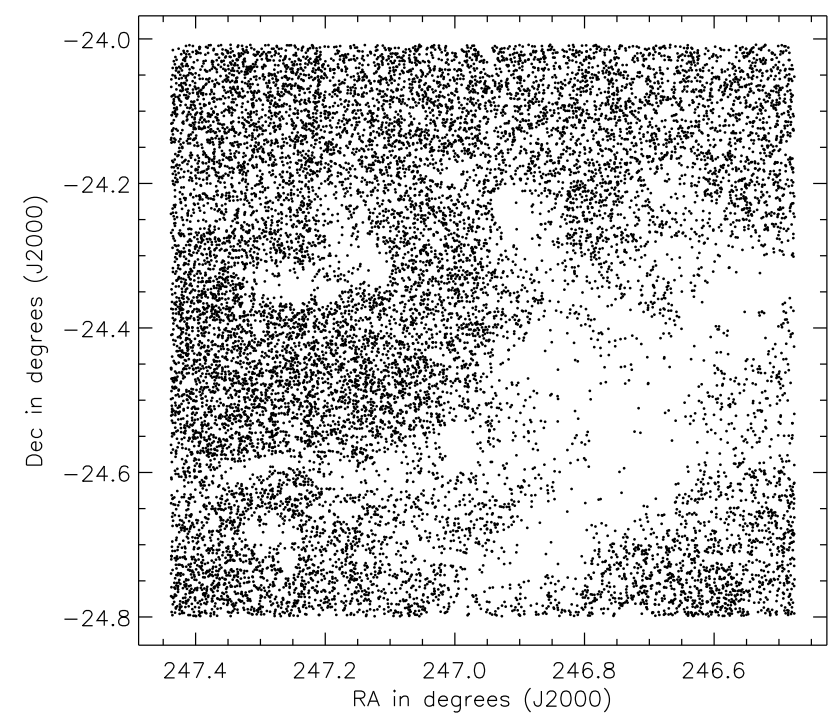

Fig. 1. Tile coverage in the $\rho$ Ophiuchi cluster. The total area covered is $\sim 0.8 \mathrm{deg}^{2}$. The spatial distribution of the detected sources reflects the cloud extinction. The visual extinction in the core is estimated to be 50-100 mag (Wilking \& Lada 1983).

be within an angular separation of $1.0^{\prime \prime}$, since the position offsets found range from $0.1^{\prime \prime}$ to $0.8^{\prime \prime}$. It was further required that each source had at least four epochs with detections in each filter. This criterion was implemented in order to reduce the probability of false transient detections and to reduce the effects of close double stars which are not resolved in all the nights, depending on seeing conditions. In total, 15191 sources were detected across the two years of observations (Fig. 1).

The internal accuracy of the photometry between nights was further improved by measuring and removing offsets, which can arise for several reasons. The photometric calibration of WFCAM data is done with 2MASS stars in the observed frames (Dye et al. 2006; Warren et al. 2007). However, some of these stars could be variable, which would have an impact on the calibration. Furthermore, for regions in the sky with high extinction, as is the case for the central core of $\rho$ Ophiuchi, the number of available 2MASS stars is reduced, which limits the photometric accuracy. Lastly, photometric offsets can also arise from small problems in flatfielding and sky subtraction from night to night. The removal of these offsets was done separately per detector and for each of the four sky pointings. Stars with magnitudes between 12.0 and 16.0 for $K$ band and 13.0 to 17.0 in $H$ band were used for the calculations, assuming that their magnitudes, in the median, do not change in time. The final offset for one detector at a given sky pointing was taken to be the average value of the differences in magnitude between the several nights and a reference night. This offset value, up to $0.03 \mathrm{mag}$, was then removed from all the sources detected in that particular detector and sky pointing.

Spitzer counterparts were obtained from the c $2 \mathrm{~d}$ IRAC pointsource catalogues of the third data delivery, applying the recommended criteria to select highly reliable samples, i.e. only considering sources which are not extended, as estimated by the source extraction, and with a quality flag of A or B in the four IRAC bands, which corresponds to a signal-to-noise ratio $\gtrsim 10$ and $\gtrsim 7$, respectively (see Evans et al. 2005, for a detailed explanation). Spitzer fluxes were converted to magnitudes using the IRAC zero magnitude flux densities defined by Reach et al. (2005).

\section{Results}

\subsection{List of near-IR variable stars}

A variable star is one which displays photometric characteristics incompatible with photometric errors. The large sample being analysed required the use of statistical tools which can quantitatively estimate the probability that the detected photometric variations are intrinsic to the star or its circumstellar material.

The first tool used to detect variability was the reduced chi-square $\chi^{2}\left(\chi_{v}^{2}\right)$ of the magnitudes, computed using:

$$
\chi_{v}^{2}=\frac{1}{v} \sum_{i=1}^{N} \frac{\left(\operatorname{mag}_{i}-\mathrm{ma} g\right)^{2}}{\sigma_{i}^{2}}
$$

where $v$ is the number of degrees of freedom, $N$ is the number of measurements and $\sigma_{i}$ is the photometric uncertainty. In this case, the statistic represents the probability that the variations result from Gaussian noise. Therefore, the reduced chi-square index entirely relies on the assumption that the noise is Gaussian, which is only approximately the case in any real set of observations in which there is variable seeing, bad pixels, detector imperfections, etc. It is also very susceptible to outlier points and does not make use of correlated changes in multiband photometry, further limiting its use.

The second tool used takes advantage of the temporal coherence of the star's light curve and also the correlation across $H$ and $K$ bands, allowing for true signals to be extracted from within the noise. The cross-correlation index (CCI) relies on simple correlation coefficients statistics and was first proposed for use in the search of variable stars by Welch \& Stetson (1993). This method, which assumes that photometric errors for two different photometry lists should be uncorrelated, is effective as long as the difference in time between observations in each frame pair (at a given epoch) is small compared with the variation period. The index is defined as:

$$
\mathrm{CCI}=\sqrt{\frac{1}{N(N-1)}} \sum_{i=1}^{N}\left(\frac{\operatorname{mag}_{i}^{H}-\operatorname{māg}}{\sigma_{H, i}}\right)\left(\frac{\operatorname{mag}_{i}^{K}-\operatorname{ma} g}{\sigma_{K, i}}\right),
$$

where $N$ is the number of measurements and $\sigma_{H, i}, \sigma_{K, i}$ are the photometric uncertainties.

A third index $\left(\mathrm{CI}_{H}, \mathrm{CI}_{K}\right)$ was computed for each band separately which takes advantage of the temporal coherence of the light curve and can be used to detect long term variations in a single band. It is defined as:

$\mathrm{CI}_{K}=\sqrt{\frac{1}{N(N-1)}} \sum_{i=1}^{N}\left(\frac{\operatorname{mag}_{i}^{K}-\mathrm{ma} g}{\sigma_{K, i}}\right)\left(\frac{\operatorname{mag}_{i+1}^{K}-\mathrm{ma} g}{\sigma_{K, i+1}}\right)$,

for $K$ band, and similarly for the $H$ band.

For each star, the $\chi_{v}^{2}, \mathrm{CCI}, \mathrm{CI}_{H}$ and $\mathrm{CI}_{K}$, were computed for each year separately. A star was classified as variable when it met one of the following criteria:

$-\chi_{v}^{2}>17.3$ in one or two bands, meaning only 1 to 2 (to be conservative) false detections in the total sample are expected;

- CCI, $\mathrm{CI}_{H}$ or $\mathrm{CI}_{K}>$ 2.0. Numerical simulations have been used to study the behaviour of the correlation indices. For 16000 stars (approximately the size of the dataset), the distribution of values for the CCI due to random chance over 7 epochs is symmetric about zero and has a maximum value of 1.8. The simulations for the individual band correlation 


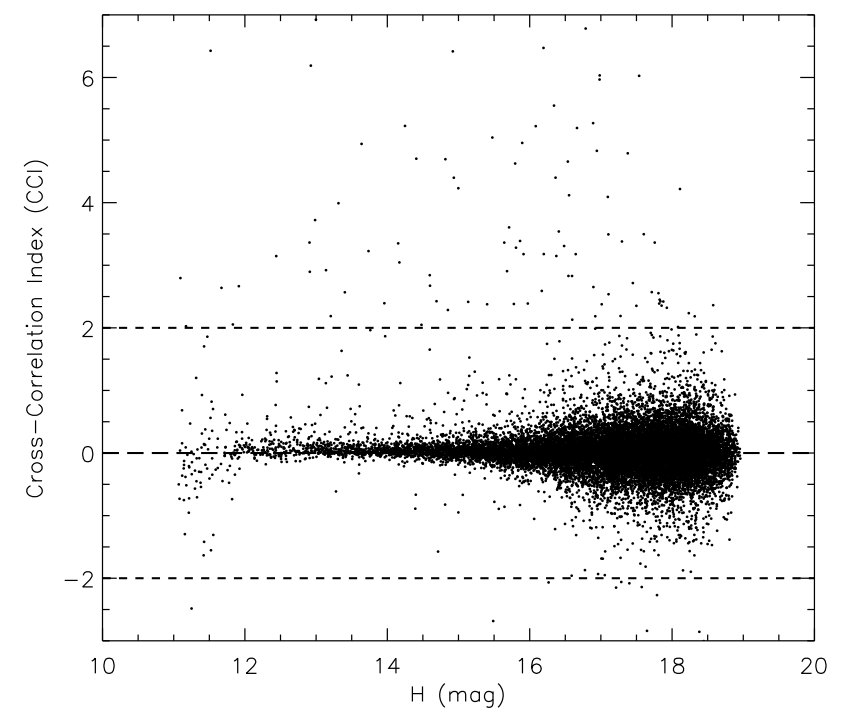

Fig. 2. Cross correlation index (CCI). Variable stars show positive values of the CCI greater than 2 .

indices showed the same behaviour, with a maximum value of the indices due to random chance of 1.9. Figure 2 shows the CCI as a function of $H$ magnitude. Non-variable stars produce a probability distribution centred about zero due to noise while true variables show positive values of the CCI greater than 2 (to be conservative). All the correlation indices showed a similar behaviour. The width of the distribution of non-variable stars depends on the total number of epochs used in the calculation of the correlation indices, and so the threshold was scaled accordingly $\left(\mathrm{CCI}, \mathrm{CI}_{H}\right.$ or $\mathrm{CI}_{K}>2,2.16,2.37,2.65$ for a star with detections in 7, 6, 5, or 4 epochs, respectively).

The application of these criteria led to a raw list of 235 candidates for variability. These were visually examined and 59 objects were rejected, primarily because they turned out to be close double stars which fell inside the aperture, showing fluctuations in magnitude which originated from differences in seeing across the different epochs. Furthermore, faint objects $(K>$ $15.5)$ which showed variability characteristics consistent with the properties of active field $\mathrm{M}$ dwarfs were also removed, since they are more likely to be affected by field contamination. Active low mass M-dwarf (dMe) stars have strong surface magnetic fields (Johns-Krull \& Valenti 1996) and show significant levels of coronal activity with maximum amplitude variations in the optical of $<0.5 \mathrm{mag}$ (Bondar' 2002). Assuming the same value of amplitude variation for IR variability (to be conservative), a simple starspot model (Vrba et al. 1986) predicts a maximum colour variation $H-K$ of $0.15 \mathrm{mag}$. All variables fainter than 15.5 in $K$-band, magnitude amplitude variations less than 0.5 , and colour variations less than 0.15 , with a $3 \sigma$ confidence, were therefore not considered in the final list of candidate members of $\rho$ Ophiuchi since they were consistent with being active $\mathrm{M}$ dwarfs. This sample could, however, contain genuine cluster members, and so a table with the main properties has been included in an appendix.

The final list contains 137 variable stars. Table 4 shows the photometric properties for each variable star, such as the coordinates, the average $H$ and $K$ magnitudes, the peak-to-peak amplitude for $H, K$, and $H-K$, the slope in the variability colour-magnitude diagram (Sect. 3.4), the SED class as determined from IRAC/Spitzer colour-colour diagrams (Sect. 3.5), several variability flags (Sect. 4.2), and references for the known members of $\rho$ Ophiuchi (Sect. 3.2).

\subsection{Known population of the Ophiuchus molecular cloud}

Many imaging and spectroscopic studies have been done on the Ophiuchus molecular cloud across the spectrum, mainly at near- and mid-infrared wavelengths (e.g., Vrba et al. 1975; Elias 1978; Wilking et al. 1989; Comeron et al. 1993; Luhman \& Rieke 1999; Bontemps et al. 2001; Allen et al. 2002; Natta et al. 2006), but also in X-rays (Gagné et al. 2004; Ozawa et al. 2005) and in the optical (e.g., Martin et al. 1998; Wilking et al. 2005). The WFCAM catalogue was merged with several catalogues of $\rho$ Ophiuchi members (namely from the following studies, Comeron et al. 1993; Bontemps et al. 2001; Gagné et al. 2004; Ozawa et al. 2005; Wilking et al. 2005), which were chosen based on their accuracy to assess membership, and also to better match the magnitude limits and spatial distribution of the WFCAM observations. WFCAM counter-parts are found for 128 previously known members of $\rho$ Ophiuchi. Of the sources not merged with these catalogues, many are present in source tables of other surveys in the literature, but they did not posses characteristics which enable them to be classified as young cloud members rather than background stars or galaxies. A significant fraction of the $\rho$ Ophiuchi population is not recovered simply because the objects are too bright, and therefore heavily saturated in the WFCAM images. Also the very extended objects are not present in the final catalogues since, to avoid galaxy contamination, this type of object was rejected. From the 128 known members with WFCAM counter-parts, $41 \%$ are variable stars, according to the previous definitions. In the sections that follow, variable stars which have been confirmed as members of the cloud by these studies are referred to as members. Variables which have not been confirmed as members are referred to as candidate members.

\subsection{Magnitudes and colours of the variable stars}

The colours and magnitudes of the variable stars are an important clue in investigating the youth and masses of these objects. The colour-magnitude diagram, $K$ versus $H-K$, shown in Fig. 3 , displays the average magnitude and colour of the variable members of $\rho$ Ophiuchi (open squares) and the candidate members (filled circles). The age of objects in the core of $\rho$ Ophiuchi has been found to be 0.3 Myr (Greene \& Meyer 1995; Luhman \& Rieke 1999) and an extensive H $\alpha$ survey by (Wilking et al. 2005) derived a median age for of 2.1 Myr, with some members up to $3 \mathrm{Myr}$, for more widely distributed members. The curves in the diagram show the model evolutionary tracks of the Lyon group (Baraffe et al. 1998; Chabrier et al. 2000; Baraffe et al. 2003) for a cluster at a distance of 119 pc (Lombardi et al. 2008). The solid curve shows the theoretical $1 \mathrm{Myr}$ isochrone, for low mass stars ranging from $0.5 M_{\odot}$ down to $0.003 M_{\odot}\left(\sim 3 M_{\text {Jup }}\right)$. The isochrone is a combination of the NextGen and the DUSTY isochrones (Baraffe et al. 1998; Chabrier et al. 2000). The dashed and dotted curves show the COND isochrones (Baraffe et al. 2003) for 1 and $5 \mathrm{Myr}$, respectively, down to $\sim 3 M_{\text {Jup }}$. The lines parallel to the redening vector (Rieke \& Lebofsky 1985) mark the separation between stars, brown dwarfs $\left(M<0.075 M_{\odot}\right)$, and planetary mass candidates $\left(M<0.012 M_{\odot}\right)$ for the different isochrones. The models indicate that the brighter candidates are above the hydrogen burning limit, but the faint sources would extend to very low masses. If an age of $1 \mathrm{Myr}$ is considered, the 


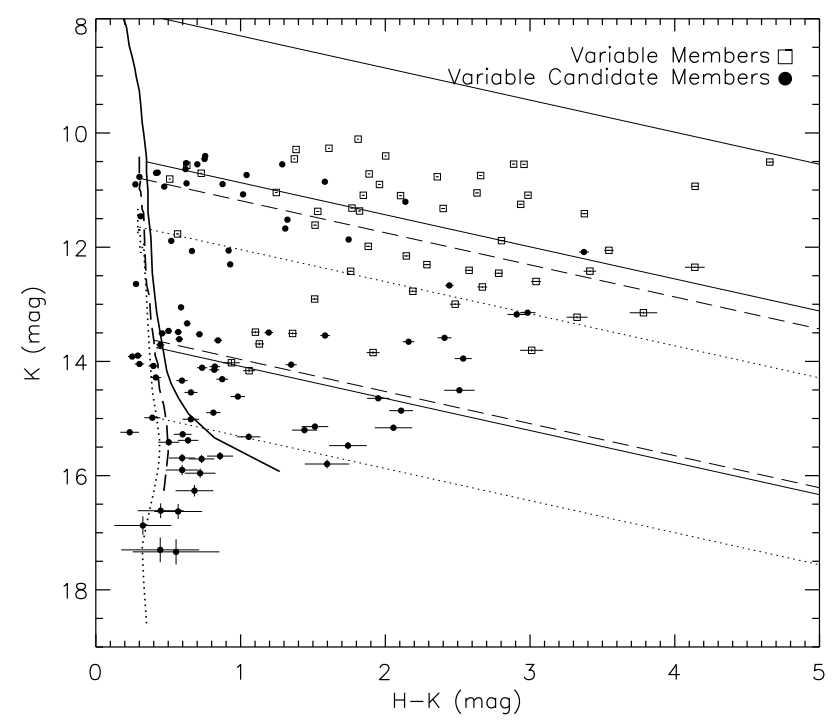

Fig. 3. Colour-magnitude diagram for variable members (open squares) and variable candidate members (filled circles) of $\rho$ Ophiuchi. Solid, dashed and dotted lines show the isochrones at $119 \mathrm{pc}$ of the Lyon group (see text for detailed explanation). Lines parallel to the redening vector (Rieke \& Lebofsky 1985) mark the separation between stars, brown dwarfs and planetary mass candidates for the different isochrones.

faintest variable objects would have just a few Jupiter masses. However, when an older age is considered (dotted line), the objects would become more massive. Wilking et al. (2005) found a distributed population in Ophiuchus which is significantly older than that in the more highly extincted cloud core, with ages up to $3 \mathrm{Myr}$, so it is plausible that part of the variable objects are older than the core population. Although the CMD gives a good indication of the approximate masses of the variable objects, a spectroscopic follow-up is needed to investigate their nature.

\subsection{Characteristics of the variability}

The timescales of variability which can be studied with this dataset can be divided into two groups: intermediate and long time scales. The first applies to the stars which only show variability in one of the two years of observations, and are likely to represent physical phenomena which have a maximum duration of a few months. The long term variables are stars that show variability behaviour across both years, which could be caused either by a single mechanism or be the result of repeated shorter term variability phenomena. In the list of member variables, $77 \%$ of the stars show variability over the two years, while $23 \%$ appear to be variable only in the first or second year. The values for the candidate members are slightly different, where $52 \%$ of the objects are variable in both years and the remaining $48 \%$ show variability in only one of the years of observations. Figure 4 is an example of a candidate member of $\rho$ Ophiuchi, which shows no significant variations in the first year but large variations in the second year. Given the sparse cadence of the observations, a search for periods has not been attempted.

The amplitude of the variations was measured as the peakto-peak fluctuation in magnitudes and colour, for each year separately and also for the combined data. Figure 5 shows histograms of the peak-to-peak magnitude amplitude for all the variable stars, members and candidate members. They show a peaked distribution, with most of the amplitude values ranging from 0.01 to $0.8 \mathrm{mag}$. Table 5 shows the mean and maximum values of the
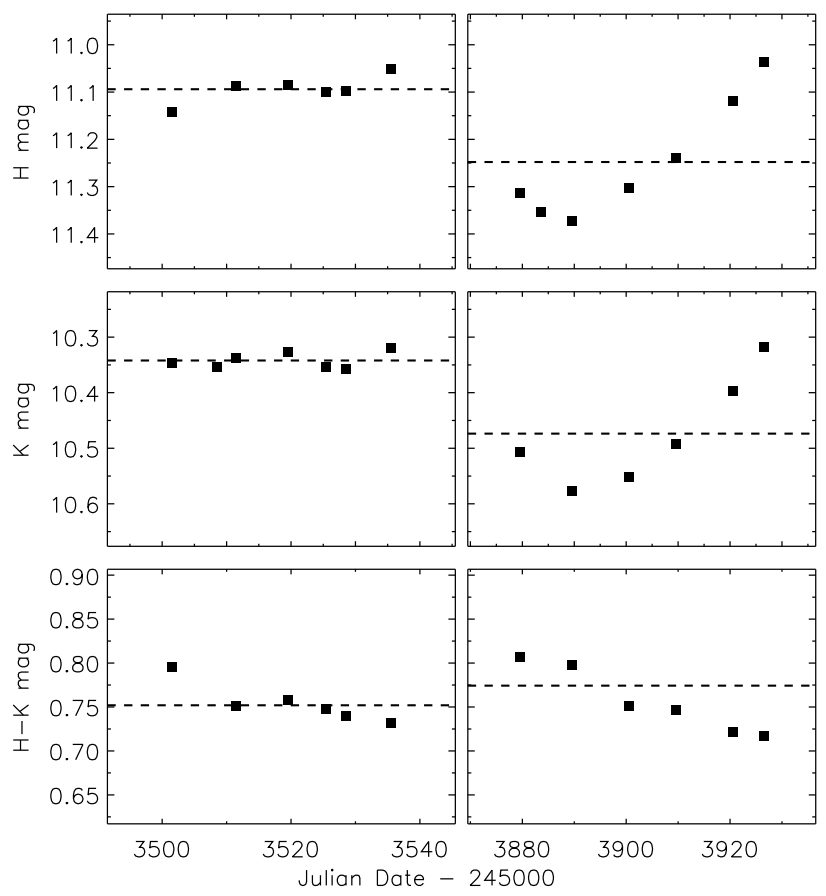

Fig. 4. AOC J162814.76-242322.5; variable candidate member star with no significant variations in the first year and large variations in the second year. The panels show the $H, K, H-K$ light curves. The left panels show the data points from 2005 May-June, and the right panels the photometry from 2006 May-July. The dashed lines show the mean values.

amplitudes for the members and candidate members. When the variation is measured across the two years, the average amplitude for the variable members is of the order of a few tenths of a magnitude. One extreme object, AOC J162636.81-241900.2, has been excluded from the calculations of Table 5 (see Sect. 4.2.5). The candidate member variables show mean $K$ and $H-K$ amplitudes of the same order of the variable members within $1 \sigma$, and a mean $H$ amplitude within $2 \sigma$. There is no statistical evidence that members and candidate members represent different populations.

Most of the variable objects show a variation in $H-K$ colour, which is on average $0.1 \mathrm{mag}$ but can be as large as 1 mag (see Fig. 6). In some cases, this variation is correlated with the change in brightness, and can be an important indicator of the underlying causes of variability (Carpenter et al. 2001). This behaviour is illustrated in Fig. 7, where the variations in the $K$-band are linearly correlated with the changes in colour. The slopes in the colour-magnitude diagram $K$ vs. $H-K$ were computed using the linear regression method for objects which showed a highly significant linear correlation between change in colour and brightness, i.e. with $<1 \%$ probability that uncorrelated variables would yield a correlation coefficient at least as high as the assumed threshold. $36 \%$ of all the variables, members and candidate members, satisfy the linear correlation criterion and the respective slopes are listed in Table 4. The angle convention used by Carpenter et al. (2001) was adopted, where a slope angle of $0^{\circ}$ represents a positive $H-K$ colour change without a variation in $K$ magnitude, and increases clockwise. Figure 8 is the histogram of the derived slopes which shows a binomial distribution with 16 objects having positive slopes, colour becoming redder as they fade, and 33 showing negative slopes, colour becomes bluer as the star gets fainter. These distinct behaviours 

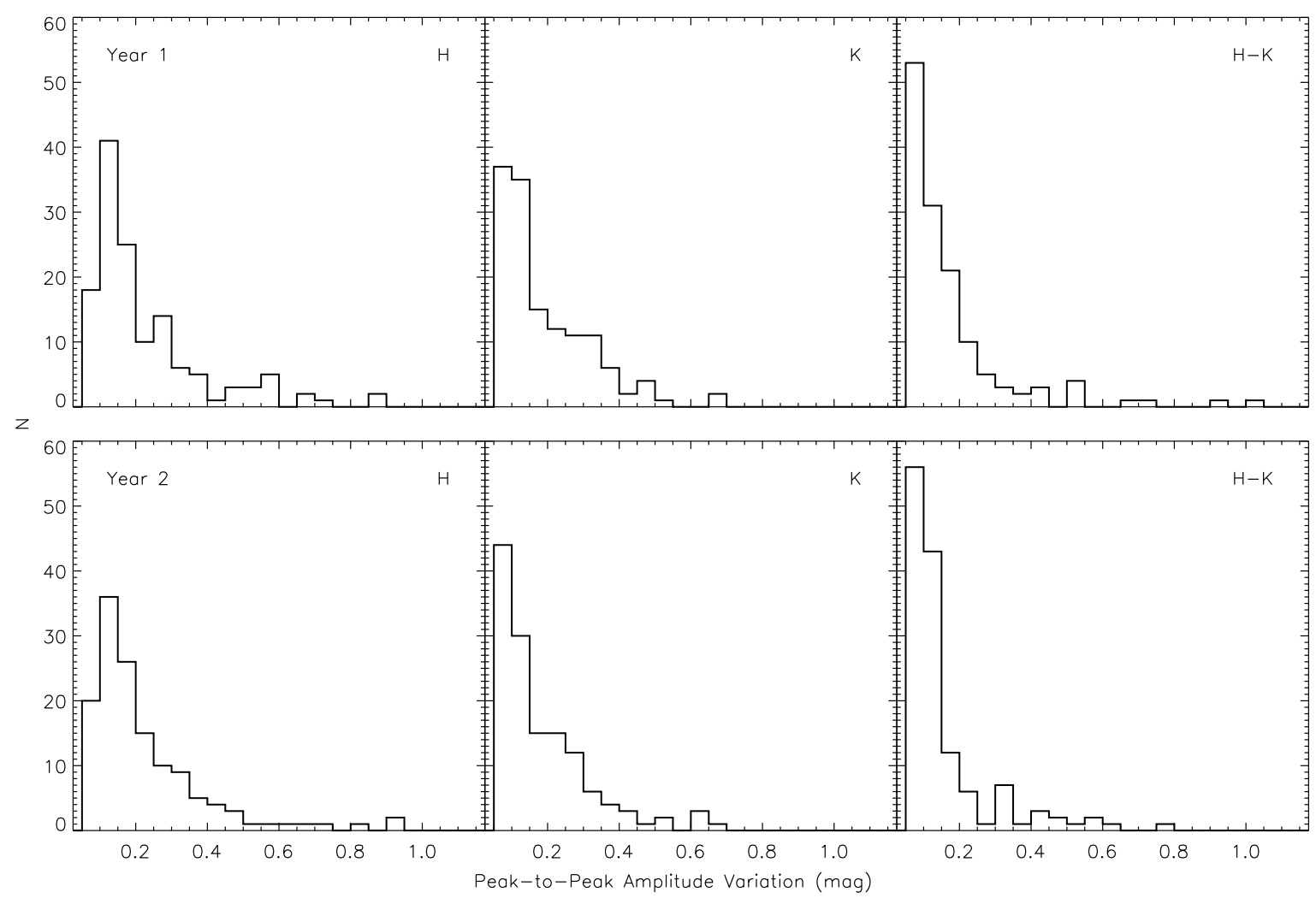

Fig. 5. Histograms of the peak-to-peak magnitude amplitude of the variations in $H, K$ and $H-K$. The top panels show the histograms for year 1 and the bottom panels for year 2 .

Table 5. Amplitudes of the variability.

\begin{tabular}{lcccccccc}
\hline \hline & & \multicolumn{2}{c}{ Year 1 } & \multicolumn{2}{c}{ Year 2 } & \multicolumn{3}{c}{ Years 1 and 2 } \\
& Band & Mean & Max & Mean & Max & Mean & $\sigma / \sqrt{n-1}$ & Max \\
\hline Variable candidates & $H$ & 0.19 & 0.82 & 0.20 & 0.88 & 0.27 & 0.03 & 0.97 \\
& $K$ & 0.15 & 0.62 & 0.15 & 0.56 & 0.21 & 0.02 & 0.62 \\
& $H-K$ & 0.09 & 0.36 & 0.08 & 0.36 & 0.15 & 0.02 & 0.52 \\
Variable candidates & $H$ & 0.17 & 0.82 & 0.15 & 0.66 & 0.21 & 0.02 & 0.82 \\
& $K$ & 0.13 & 0.61 & 0.13 & 0.61 & 0.18 & 0.02 & 0.65 \\
& $H-K$ & 0.14 & 0.97 & 0.12 & 0.70 & 0.18 & 0.02 & 0.97 \\
\hline
\end{tabular}

can be associated with physical processes and will be discussed later in this paper (see Sect. 4.2).

\subsection{Infrared excesses}

Young stars show infrared emission which originates from dusty envelopes and circumstellar discs surrounding the central object. Lada \& Wilking (1984), based on the level of long wavelength excess with respect to a stellar photosphere emission, identified three different classes which define an IR-excess or SED classification scheme: Class I, low mass protostars surrounded by an infalling envelope with large IR-excess; Class II, young stars with accretion discs and a moderate IR-excess (as classical T Tauri stars, CTTSs); and Class III, stars which no longer accrete matter from a circumstellar disc and show no IR-excess (as weak-line T Tauri stars, WTTSs). The IRAC data from Spitzer allows the study of these objects in the mid-IR, where the excess contribution from discs and envelopes is predominant. The IRAC colour-colour diagram ([3.6]-[4.5] vs. [5.8]-[8.0]) was presented as a tool to separate young stars of different classes (Allen et al. 2004; Megeath et al. 2004), and was already used in the study of other star forming regions as, for example, Taurus
(Hartmann et al. 2005; Luhman et al. 2006), Serpens (Harvey et al. 2007), or Chamaeleon II (Porras et al. 2007).

The majority of the known population of $\rho$ Ophiuchi has been classified into SED classes. Bontemps et al. (2001) used ISOCAM mid-IR bands ( 6.7 and $15.3 \mu \mathrm{m})$ to detect and classify 212 sources. Using ground-base mid-IR observations, Barsony et al. (2005) confirmed those results. Also in the X-ray regime, Chandra and XMM-Newton observations (Imanishi et al. 2001; Gagné et al. 2004; Ozawa et al. 2005) have contributed to the classification. The variability catalogue contains 48 variable members of $\rho$ Ophiuchi which were classified in these studies into Class I, II, or III. Figure 9 shows the IRAC colour-colour diagram for all variable objects with detections in the four bands, where the top diagram displays $\rho$ Ophiuchi members and the bottom diagram the candidate members. The variable members are displayed according to the SED class assigned in the literature, i.e., Class I (open squares), Class II (crosses), and Class III (open triangles). The extinction vector is from Flaherty et al. (2007). For comparison, a sample of objects has been chosen from a region of the sky away from the central cloud with little extinction which should mainly contain field stars. 

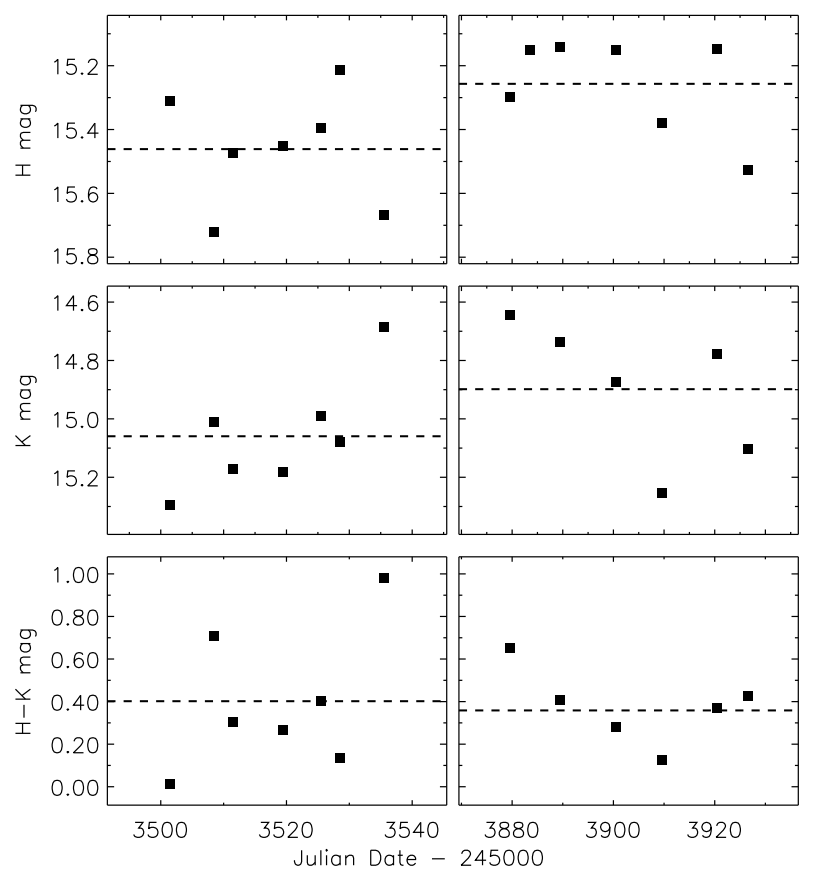

Fig. 6. AOC J162814.72-242846.5; variable star with large variations in magnitude as well as in colour.

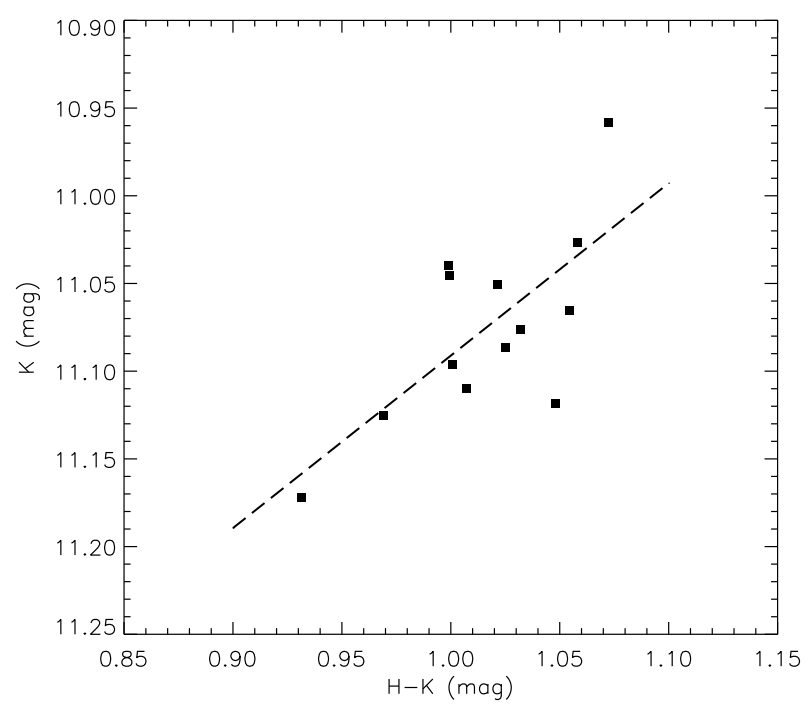

Fig. 7. AOC J162812.71-241135.7; variable star with linearly correlated changes in $H-K$ colour and brightness in $K$ band. Its colour gets bluer as the star gets fainter. The dashed line shows the linear fit.

The objects tend to cluster around three main regions of the diagram. Centred in the origin, [3.6]-[4.5], [5.8]-[8.0] $=(0,0)$, are sources which have colours consistent with stellar photospheres and have no intrinsic IR-excess. These can be foreground and background stars, but also Class III stars which do not have significant circumstellar dust. In this region of the colour-colour plane, it is not possible to differentiate between young stars and contaminants. However, as it can be seen in the top panel of the diagram, many of these objects are confirmed young members of $\rho$ Ophiuchi both from mid-IR and X-ray studies. Another preferred region for objects in the diagram is located within the box defined by Allen et al. (2004) which represents the colours expected from models of discs around young, low-mass stars. In fact, many of the Class II objects classified by Bontemps et al. (2001) lie within that range. However, some

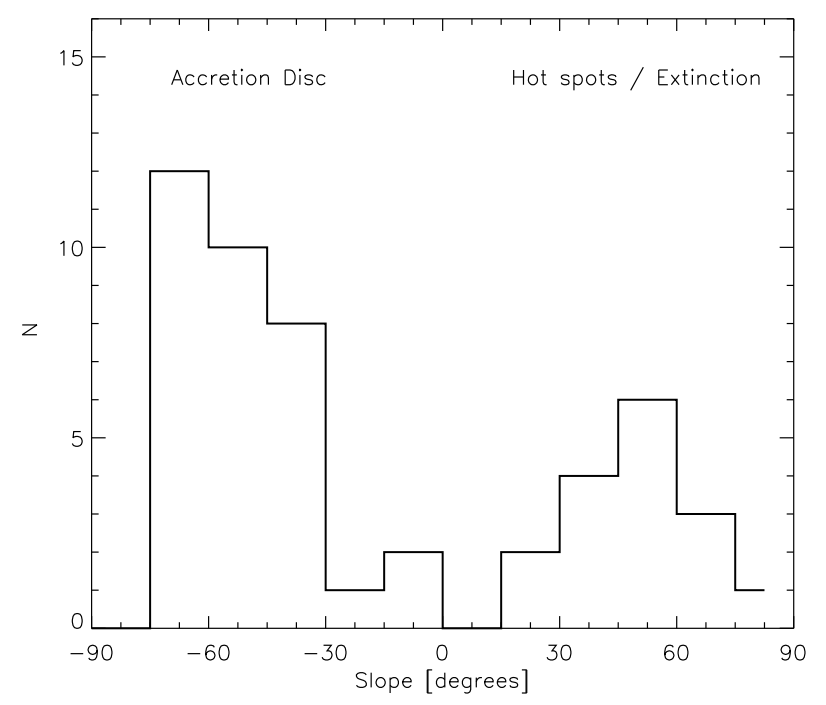

Fig. 8. Histogram of the derived slopes in the $K$ vs. $H-K$ diagram. The slope can be used as a tool to distinguish the physical mechanisms behind the observed variations (Sect. 4.2).

sources previously identified as Class II do not fall within the predicted limits. These objects have colours inconsistent with Class II sources (higher [3.6]-[4.5] colours than Class II but lower [5.8]-[8.0] colours than Class I) and were previously classified as candidate flat spectrum objects. Their location in this diagram does not confirm them as transition objects between Class I and II (Bontemps et al. 2001) since they can be explained as reddened Class II sources. Finally, from models of infalling envelopes, Allen et al. (2004) predicts the colours of Class I sources to have $([3.6]-[4.5])>0.8$ and/or $([5.8]-[8.0])>1.1$, which agrees well with the two Class I objects identified with ISOCAM (Bontemps et al. 2001). Likewise, one of the sources classified with ISOCAM as Class III lies on the Class I/reddened Class II region of the colour-colour diagram. In the ISOCAM paper, this source was classified as Class III because it is located within the CS contours of the cloud, as defined by Liseau et al. (1995). According to the IRAC diagram, its previous classification is incorrect.

From the 84 candidate members, 37 are detected in the four IRAC bands and plotted in the bottom panel of Fig. 9 (filled circles). Four variable members are also detected in the four band but have no assigned SED class and therefore are also included (open diamonds). According to the criteria described above, they can be divided into Class I ( 2 objects), Class II (11 objects), and possibly Class III (27 objects).

The number of variables with detections in the $4.5 \mu \mathrm{m}$ IRAC/Spitzer band is higher than for longer wavelengths, since this band has a higher sensitivity. Figure 10 shows the $H-K$ vs. $K-[4.5]$ colour-colour diagram for all variable objects with detections in the IRAC/Spitzer $4.5 \mu \mathrm{m}$ band, where again the top diagram displays the members of $\rho$ Ophiuchi and the bottom diagram the candidate members. The variable members are displayed according to their SED class from the literature (same convention as in Fig. 9). The reddening vector is from Flaherty et al. (2007) and the dash line follows the same reddening law. The top panel of Fig. 10 shows a clean break between Class I/II and Class III stars (i.e., stars with and without circumstellar dust emission) for $K-$ [4.5] $>1$ and $H-K>0.5$. Applying this criterion to the variable candidate members (bottom panel), 23 candidate members can be classified as ClassI/II, and 8 as possible Class III. Furthermore, for objects present in both Figs. 9 and 10, 


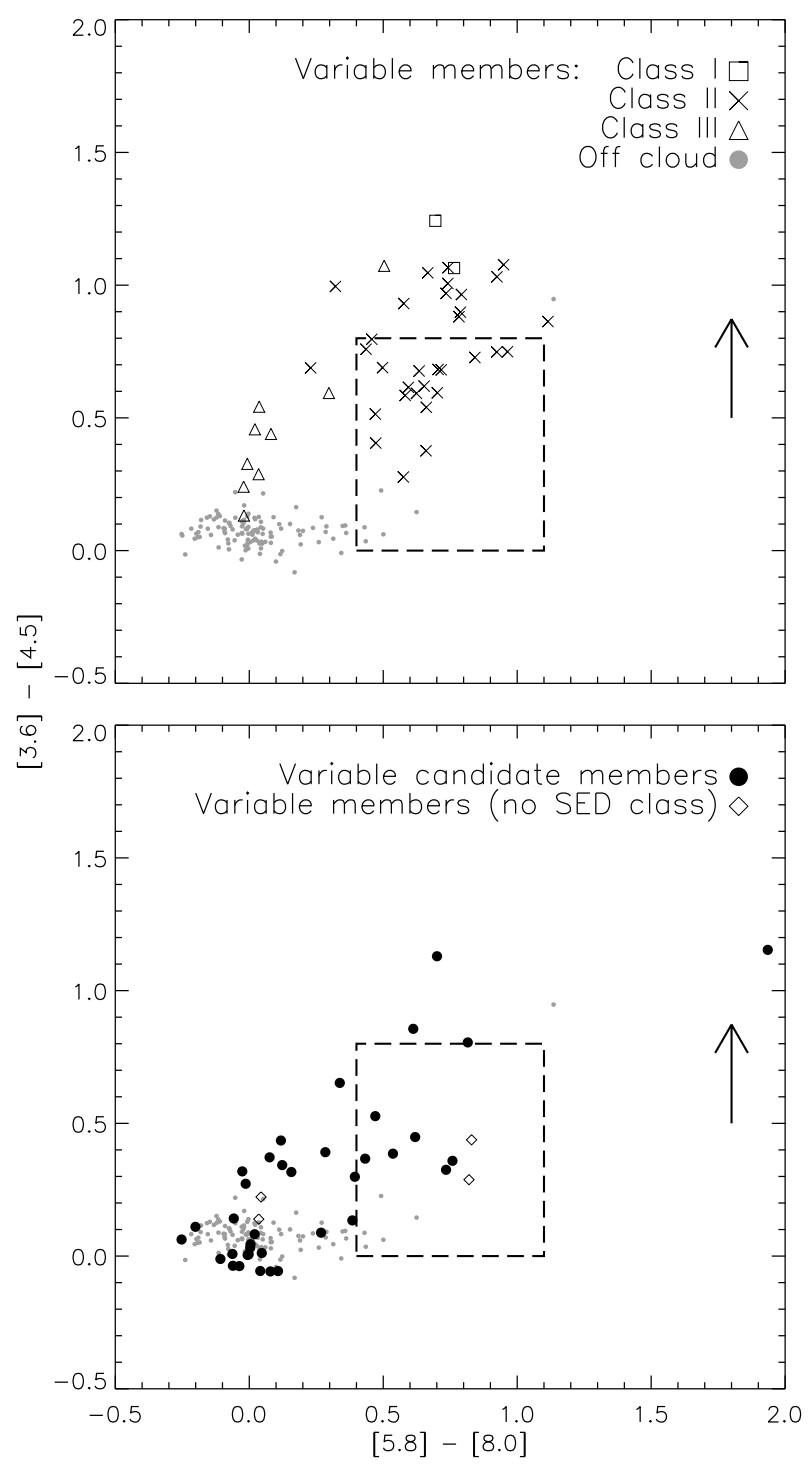

Fig. 9. IRAC colour-colour diagram for variable members (top panel) and candidate members (bottom panel). Arrow represents the $A_{K}=4$ extinction vector for $\rho$ Ophiuchi (Flaherty et al. 2007).

the SED classes determined are in agreement. The only exception are 3 variable candidate members which show a significant IR-excess in the $K-[4.5]$ colour but less at longer wavelengths.

Combining WFCAM near-IR observations with IRAC/Spitzer mid-IR data, it is possible to classify $60 \%$ of the candidate members as Class I/II and possible Class III. Furthermore, the SED classes from the literature for the variable members are confirmed, with the exception of few objects (see discussion above).

\section{Discussion}

\subsection{Membership}

The nature of the candidate member population is very important, since it determines the reliability of variability as an indicator of youth. In principle, some or all of the candidate members could be background objects - either variable stars within our own galaxy or extragalactic variable objects, principally Active Galactic Nuclei (AGN). An inspection of Fig. 11, however, shows that this is unlikely to be the case. The candidate

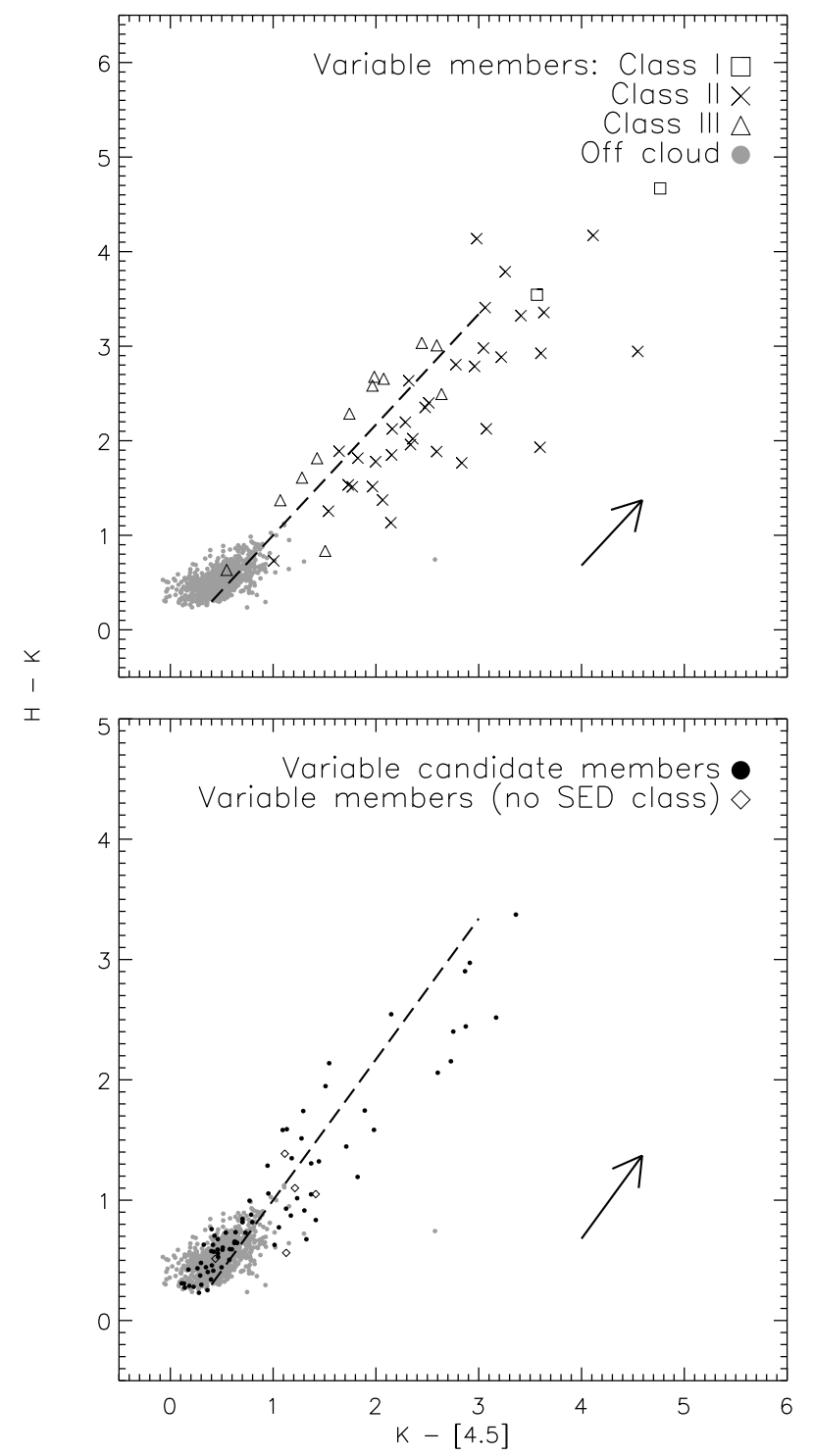

Fig. 10. WFCAM/IRAC colour-colour diagram. Arrow represents the $A_{V}=10$ extinction vector for $\rho$ Ophiuchi (Flaherty et al. 2007).

members do not appear to be distributed in the same way as the majority of background point sources against which the clouds can be seen - they are noticeably absent from the regions of lowest extinction for example, and appear to be more clustered. To put this on a quantitative basis the 2-D Kolmogorov-Smirnov (K-S) test as outlined by Peacock (1983) was applied to candidate member and full point-source detection (non-variable) samples - the latter clearly dominated by the background population of stars and galaxies. The test is approximately distribution-free, which means the probability that the two samples are from the same population can be estimated. For 84 candidate member objects, a $Z$ statistic of 1.99 was determined against the background distribution. Peacock (1983) gives a formula for calculating the significance level, which shows that there is an $2 \%$ chance that the two are from the same population. This is an upper limit since, for reasons already discussed, the variable candidate members lie outside the regions of high extinction, as do the background sources. This introduces an unavoidable spatial bias, making the two distributions look similar. The K-S test suggests the two populations come from different distributions. Of course this is for the candidate population as a whole - there is 


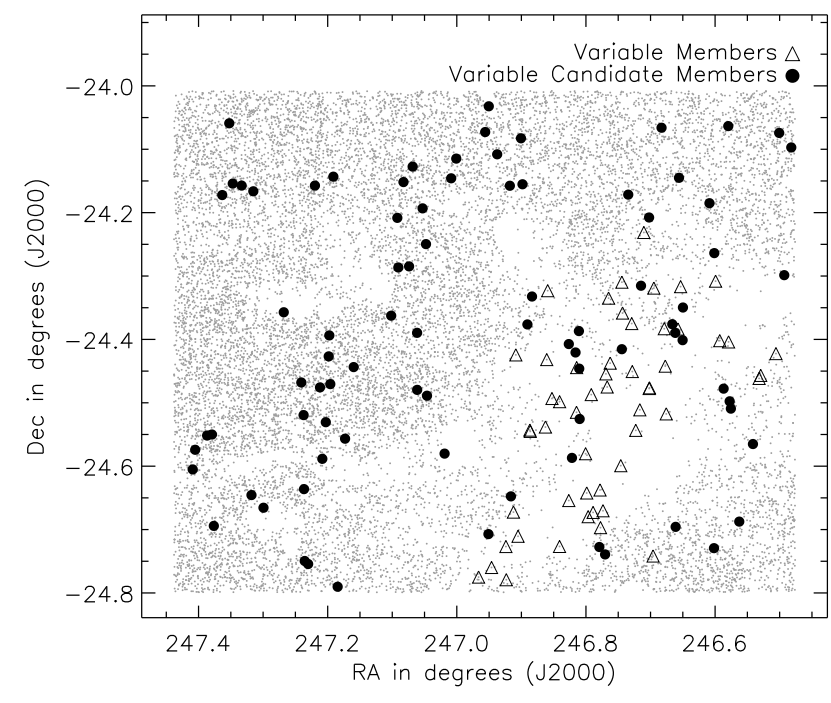

Fig. 11. Spatial distribution for variable members of $\rho$ Ophiuchi and variable candidates, with respect to all detected objects in the field.

still the possibility that a subset are due to contamination from background variable objects.

AGN are a potentially important source of contamination given the faint magnitude limits of the sample. The $K=$ 18.0 limit amounts to $0.08 \mathrm{mJy}$ at $3.6 \mu \mathrm{m}$ assuming an SED median index of -1.2 (Buchanan et al. 2006). Treister et al. (2006) use a Spitzer survey of the GOODS field to estimate AGN number density versus flux in the IRAC bands. Their data show that this flux limit should result in approximately 300 AGN detections per sq. degree. However, central to understanding contamination by AGN is the distribution and magnitude of extinction in $\rho$ Ophiuchi. Kenyon et al. (1998), in a study of the reddening law, find that in the clear areas well outside the molecular gas contours in areas of least extinction, the $H-K$ colour in most sources has only a small excess over intrinsic values - of order 0.2 in $H-K$. A similar excess is found in these data, corresponding to a visual extinction of 3-4 mag. However, over much of the field the extinction is much higher. Therefore, for a proper evaluation, the number counts of $K$-band point sources (Fig. 1) was used to calculate $K$ band extinction in a grid of $10 \times 10$ squares over the observed field. This was then used with the AGN cumulative flux distribution (Treister et al. 2006) to give the integrated number of AGN expected over the WFCAM field of view. After integrating over the extinction distribution a total of 33 AGN should be present in the $\rho$ Ophiuchi field to the $K$ band detection limit of 18.0. How many of these will be detected as variable? With the steep AGN cumulative flux distribution, more than $95 \%$ of the AGNs will lie in the faintest two magnitude bins, where the typical photometric error of the sample is $0.05-0.1 \mathrm{mag}$. Simulations with the 2-band cross-correlation index show that a clear variability detection with this index requires an amplitude of at least $>0.13 \mathrm{mag}$ in this case. Enya et al. (2002) show that the fraction of AGN with long-term IR variability larger than this is $28 \%$ or 9 objects in the $\rho$ Ophiuchi field, compared to the candidate-member sample of 84 . So while a precise number is difficult to calculate, it is likely that a small (but still non-negligible) fraction of the variable candidate-members can be explained as AGN.

Another potential source of contamination is a population of background variable stars. The galactic model of Wainscoat et al. (1992) was used as a useful tool in investigating this. Even though discrepancies between observations and this model exist, it is certainly accurate enough (factor of 2) for the purposes here. Schultheis et al. (2000) conducted an unbiased survey of a region in the galactic bulge, and found 720 sources variable in $J$ and $K$ band, representing $0.2 \%$ of the source detections in the region. These were mainly long-period variables - AGB stars at or beyond the tip of the RGB. A similar fraction of variables in the $\rho$ Ophiuchi field (15 191 sources) would predict 24 variables. However, the stellar populations are very different in the two fields. The bulge field is dominated by giants and includes many AGB stars; in fact the galactic model predicts a number of AGB stars comparable to the total number of variables detected in the Schultheis et al. (2000) study. But the high galactic latitude of the $\rho$ Ophiuchi field $(+16.7)$ completely changes the result. Far fewer giants are expected, since the line of sight moves rapidly above the plane and bulge, so that the counts become dominated by the faint end of the dwarf luminosity function. At this latitude, the model predicts $<1$ AGB star/long-period variable in the whole field of view. It seems clear that the $\rho$ Ophiuchi variable sample should be completely uncontaminated by these objects.

It should be noted that in discussing the membership of any individual source, its location in the field is important, since any significant amount of extinction lowers the probability of background contamination substantially compared to the numbers calculated above.

\subsection{Understanding the variability}

The characteristics of the variability observed in young stars in $\rho$ Ophiuchi are very diverse, spanning large ranges in magnitude, colour and timescale. Furthermore, given the limited information and finite measurement errors, it is not possible to conclude with certainty which physical mechanism is causing variability in each star. In fact, in many cases the variability observed in a particular star could be caused by a variety of mechanisms. So the following approach was used. Known or plausible physical mechanisms were considered in turn as the cause of variability in each star. Mechanisms which were considered plausible according to certain criteria were then listed in Table 4 for each star. In this way, an overall idea of whether the variability can be explained by conventional mechanisms, is obtained. A summary of the mechanisms, criteria and conclusions is as follows.

\subsubsection{Rotational modulation by cool starspots}

Cool starspots are known to exist both in CTTSs and WTTSs, and to cause variations in the stellar brightness. They are colder than the photosphere and arise from magnetic active regions, analogously to solar sunspots (Bouvier \& Bertout 1989). The fractional area coverage is of the order of $20 \%$ (Bouvier et al. 1993; Herbst et al. 1994) which determines the maximum amplitude of 0.4 mag (Carpenter et al. 2001; Herbst et al. 2002). Herbst et al. (1994) presented a classification scheme for optical variability, defining as type I variable stars with low, periodic, amplitude magnitude variations (a few tenths of a magnitude) caused by cool spots. Photometric variability studies of very low mass stars and brown dwarfs have identified similar behaviours (e.g., Scholz \& Eislöffel 2005). Using single-temperature blackbody models and input parameters inferred from optical studies to model stellar spots behaviours, Carpenter et al. (2001) find that the expected amplitude colour variations produced by cool spots do not exceed 0.05 mag both in $J-H$ and $H-K$ colours. 
In the $\rho$ Ophiuchi variable population, stars with amplitudes in $H$ and $K<0.4$ mag and $H-K$ colour variations $<0.05$ mag show variations consistent with the existence of cool spots, as defined by the above parameters, and are classified as CS (cool spots; see Col. 7, Table 4). These amount to $17 \%$ of the variable members and $21 \%$ of the candidate members. Late-type field stars with magnetic activity could, however, still contaminate this group of variables (Sect. 3.1).

\subsubsection{Rotational modulation by hot starspots}

Hot spots are interpreted as the impact points on the stellar surface from disc accretion through magnetic field lines (e.g., Calvet \& Hartmann 1992). They cover a smaller fraction of the stellar surface but the high temperatures can cause larger amplitude variations, which in the near-IR regime can be as high as $0.2-0.4$ mag for the $J H K$ bands, and between $0.05-0.12$ in colours (Carpenter et al. 2001). The timescales are shorter compared to cool spots (e.g., Kenyon et al. 1994). In the Herbst et al. (1994) classification scheme, a type II variability class is assigned to objects showing larger amplitude variations (which can be irregular or periodic), from short-lived hot spots. To analyse the behaviour produced by hot spots, Carpenter et al. (2001) use the information from the correlated colour and magnitude changes. The predicted slope from hot spot models has high, positive values, although given the simplicity of their models, it is not possible to identify a specific range.

Variable stars with amplitudes in $H$ and $K<0.4$ mag, $H$ and $K$ colour variations $0.05-0.12 \mathrm{mag}$, and a positive slope in the colour-magnitude diagram (if a slope was determined) are classified as HS (hot spots). Hot spots can explain variability in $28 \%$ of the variable members and $30 \%$ of the candidate members.

\subsubsection{Circumstellar extinction}

Variations in extinction can originate from the intersection with the line-of-sight of infalling or orbiting material in the circumstellar environment (Skrutskie et al. 1996). Circumstellar extinction variations will follow a reddening law if the grain size of the material is comparable to the interstellar grain size distribution (Skrutskie et al. 1996). The interstellar reddening law $E(H-$ $K) / A_{K}=0.56$ was adopted from Rieke \& Lebofsky (1985). In the $K$ vs. $H-K$ diagram, this means that extinction variations can explain photometric slopes of $60^{\circ}$, with the object being redder when it is faint. The histogram of the colour-magnitude slopes (Fig. 6) peaks at $45^{\circ}-60^{\circ}$, showing that variations in extinction can explain most of the observed positive slopes.

Variable stars with positive slopes between $45^{\circ}$ and $60^{\circ}$ are classified as E (extinction). These represent $6 \%$ of the variable members and $4 \%$ of the variable candidate members.

\subsubsection{Accretion discs}

Changes in the disc structure caused by mechanisms such as thermal instabilities, variable magnetic fields or warping instability can lead to near-IR variations on short timescales (see Carpenter et al. 2001, and references therein). Using simple disc models to study the consequences expected from geometric variations in a circumstellar disc, Carpenter et al. (2001) find that the observed negative slope in the colour-magnitude correlation (Fig. 5) can be explained by changes in the inner disc structure. This scenario has also been suggested by Eiroa et al. (2002).

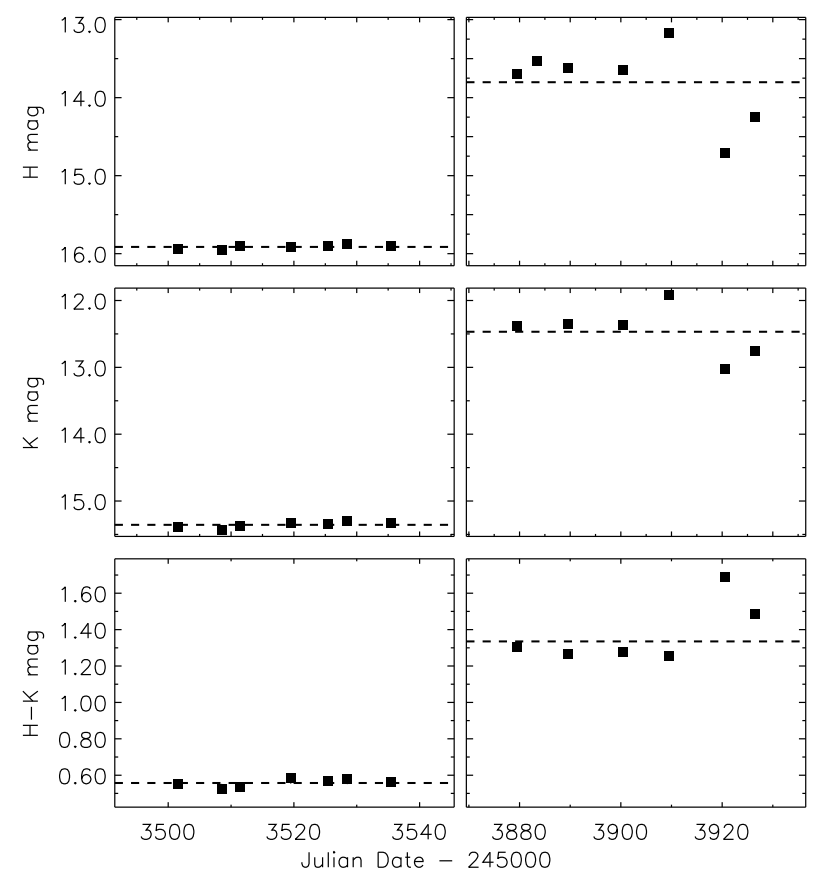

Fig. 12. AOC J162636.81-241900.2: variable member with a brightening of several magnitudes in both $H$ and $K$ bands.

Variable stars for which a negative colour-magnitude slope was found, where the star is bluer when faint, are classified as AD (accretion disc variations). In $\rho$ Ophiuchi, 23\% of the variable members and $25 \%$ of the variable candidate members have a negative slope.

\subsubsection{Extreme variability}

Although very rarely observed, another possible cause of variability related to accretion discs, is an $\mathrm{Fu}$ Orionis type outburst (e.g. Reipurth et al. 2007, and references therein). AOC J162636.81-241900.2 (Fig. 11) has been previously classified as a member of $\rho$ Ophiuchi. Its light curve reveals a brightening of several magnitudes (3.5 mag in the $K$-band) in approximately one year, in what could be a $\mathrm{Fu}$ Orionis type burst. However, a minimum in $H$ and $K$ magnitudes of 13.93 and 12.31 , respectively, have previously been reported by Barsony et al. (1998), meaning this object could instead be an EXor, which are stars that undergo a maximum in magnitude once every few years, thought to be caused by the massive infall of circumstellar material onto the central star (see Herbig 2008, and references therein). A spectroscopy study of this variable member (classified as EV) is needed to better understand its nature.

\subsubsection{Unexplained variability}

There are $32 \%$ of the members and $26 \%$ of the candidate members which do not fit into any of the criteria defined above. Among the members, 15 objects out of 17 have colours consistent with SED Class I/II stars. However the majority does not show a linear correlation in the colour-magnitude variability diagram. The most likely explanation is that several physical processes are taking place simultaneously, and the variability observed is the combination of all the intervenients. This scenario is further supported by the fact that 4 of these variable members show a positive slope which is either too shallow or 
to steep to be explained by changes in extinction, and colours inconsistent with hot spots rotational modulation. The same is true for the only candidate member with a determined, positive slope, which has also been classified as Class II, but does not fit into the variability categories. All the other candidate members with unexplained variability did not show a linear correlation in the colour-magnitude variability diagram, and very few have IRAC/Spitzer detections. The other characteristic which is valid for these objects, both members and candidate members, is that the mean $H$ and $K$ amplitude magnitudes, as well as the $H-K$ colour variations, are twice as large as the ones seen among other variables. Although this is in conformity with the scenario just described, it also opens the possibility that more extreme variability behaviours are, after all, common among young stars. Only targeted photometric and spectroscopic monitoring of variable stars of different types, looking for a correlation between colours, magnitudes, and spectral features would help in isolating the important physical mechanisms.

\section{Conclusions}

A multi-epoch, very deep near-IR survey was conducted in the Ophiuchus molecular cloud with the WFCAM/UKIRT. Statistical methods, such as the reduced chi-square and correlation indices, were used in the search for variability, known to be a defining characteristic of young stellar objects. 137 variable objects were found which show timescales of variation which can go from days to years, amplitude magnitude changes from a few tenths to $3 \mathrm{mag}$, and significant colour variations. From the $\rho$ Ophiuchi known population, 128 members have counter-parts in the WFCAM catalogue, and $41 \%$ are found to be variable.

The dataset was merged with IRAC/Spitzer observations to further extend the information about the detected variables and using colour-colour diagrams, ([3.6]-[4.5] vs. [5.8]-[8.0]) and/or $(H-K$ vs. $K-[4.5]), 50$ variable candidate members were classified as candidate pre-main-sequence stars (23 Class I/II and 27 possible Class III variables).

The variability trends found were interpreted in a physical context by comparison with previous suggested classifications for variability in the optical and near-IR. The characteristics observed are consistent with the existence of cool or/and hot spots on the stellar surface, variations in circumstellar extinction, or structural variations in accretion discs. However, a large fraction of the variable population does not fit into the predicted parameters for near-IR variability, which can be explained by the fact that the variability observed does not reflect a single event, but the net effect of several simultaneous processes.

Finally, near-IR variability was used to discover a candidate population of pre-main-sequence stars associated with the $\rho$ Ophiuchi star forming region. The colour-magnitude diagram, $K$ vs. $H-K$, provides evidence that many of the newly found low-luminosity variables have colours consistent with those expected from young, very low mass objects. To confirm the nature of these variable objects, a low resolution near-IR spectroscopic follow-up has been conducted for a small sample and results will be present in an accompanying paper.

Acknowledgements. We thank the UKIRT observatory staff and WFCAM Science Archive team for acquiring and pipeline processing the observations used for this project. C.A.O. acknowledges partial support from a Marie Curie Fellowship for Early Research Training. This work is based in part on observations made with the Spitzer Space Telescope, which is operated by the Jet Propulsion Laboratory, California Institute of Technology under a contract with NASA.

\section{Appendix A: Other variable stars}

Table A.1. List of variable objects with variability characteristics consistent with active field $\mathrm{M}$ dwarfs. The list could still contain young very low mass objects, possibly members of Ophiuchus.

\begin{tabular}{|c|c|c|c|c|c|}
\hline AOC designation & $\begin{array}{c}\langle H\rangle \\
\text { (mag) }\end{array}$ & $\begin{array}{c}\langle K\rangle \\
\text { (mag) }\end{array}$ & $\begin{array}{c}\Delta H \\
\text { (mag) }\end{array}$ & $\begin{array}{c}\Delta K \\
(\mathrm{mag})\end{array}$ & $\begin{array}{c}\Delta(H-K) \\
(\mathrm{mag})\end{array}$ \\
\hline J162554.64-244112.8 & 18.20 & 17.70 & 0.51 & 0.33 & 0.52 \\
\hline J162556.76-243001.1 & 17.88 & 16.30 & 0.22 & 0.23 & 0.21 \\
\hline J162609.95-240046.7 & 17.22 & 16.84 & 0.10 & 0.27 & 0.32 \\
\hline J162611.05-241208.8 & 17.66 & 17.18 & 0.19 & 0.40 & 0.37 \\
\hline J162612.86-241244.1 & 17.71 & 17.01 & 0.22 & 0.29 & 0.16 \\
\hline J162619.42-243233.8 & 18.38 & 16.98 & 0.35 & 0.31 & 0.32 \\
\hline $\mathrm{J} 162621.54-241242.5$ & 18.61 & 17.80 & 0.60 & 0.32 & 0.48 \\
\hline J162623.33-242351.4 & 18.09 & 16.47 & 0.57 & 0.30 & 0.52 \\
\hline J162631.14-241208.5 & 16.91 & 15.65 & 0.12 & 0.11 & 0.12 \\
\hline J162647.88-240946.8 & 16.42 & 15.64 & 0.27 & 0.26 & 0.16 \\
\hline J162700.96-241230.6 & 18.38 & 17.72 & 0.40 & 0.30 & 0.48 \\
\hline J162705.45-240519.9 & 17.02 & 16.43 & 0.20 & 0.15 & 0.13 \\
\hline J162749.40-242919.3 & 17.82 & 16.61 & 0.36 & 0.20 & 0.41 \\
\hline J162749.80-243950.1 & 16.94 & 16.02 & 0.39 & 0.28 & 0.18 \\
\hline J162750.41-244042.7 & 17.36 & 16.43 & 0.27 & 0.07 & 0.26 \\
\hline J162800.15-241222.8 & 18.35 & 17.25 & 0.58 & 0.16 & 0.53 \\
\hline J162801.81-241305.0 & 17.32 & 16.44 & 0.25 & 0.12 & 0.33 \\
\hline J162803.22-240536.7 & 16.18 & 15.63 & 0.32 & 0.20 & 0.15 \\
\hline J162814.41-244546.2 & 16.88 & 16.49 & 0.21 & 0.18 & 0.08 \\
\hline J162818.95-241319.1 & 17.31 & 16.68 & 0.20 & 0.26 & 0.15 \\
\hline J162840.66-243148.3 & 17.72 & 17.22 & 0.42 & 0.32 & 0.35 \\
\hline J162841.89-242705.6 & 16.33 & 16.02 & 0.18 & 0.20 & 0.16 \\
\hline J162844.20-240115.1 & 17.75 & 17.21 & 0.32 & 0.28 & 0.33 \\
\hline J162846.90-240212.9 & 16.82 & 16.46 & 0.08 & 0.32 & 0.28 \\
\hline J162852.34-242849.1 & 17.63 & 17.37 & 0.25 & 0.25 & 0.34 \\
\hline J162858.30-243544.2 & 17.50 & 16.60 & 0.22 & 0.17 & 0.08 \\
\hline J162902.71-242837.7 & 16.12 & 15.78 & 0.21 & 0.13 & 0.21 \\
\hline J162905.65-242314.9 & 17.79 & 17.50 & 0.22 & 0.20 & 0.36 \\
\hline J162911.02-242240.0 & 17.88 & 17.50 & 0.32 & 0.20 & 0.22 \\
\hline J162919.04-241038.8 & 17.72 & 17.15 & 0.27 & 0.24 & 0.22 \\
\hline J162920.93-241936.8 & 16.36 & 15.90 & 0.14 & 0.11 & 0.11 \\
\hline J162929.44-243426.3 & 18.00 & 17.65 & 0.57 & 0.43 & 0.59 \\
\hline J162930.59-241907.8 & 18.21 & 17.68 & 0.42 & 0.43 & 0.28 \\
\hline J162933.26-243359.0 & 17.96 & 17.42 & 0.47 & 0.27 & 0.59 \\
\hline J162937.02-243035.2 & 16.75 & 16.40 & 0.13 & 0.19 & 0.10 \\
\hline J162939.40-242309.4 & 17.19 & 16.82 & 0.30 & 0.19 & 0.21 \\
\hline J162943.28-243056.4 & 17.96 & 17.57 & 0.54 & 0.52 & 0.38 \\
\hline J162943.49-243102.7 & 16.67 & 16.45 & 0.22 & 0.18 & 0.23 \\
\hline J162945.00-243421.7 & 17.71 & 17.39 & 0.46 & 0.33 & 0.28 \\
\hline
\end{tabular}

\section{References}

Ali, B., \& Depoy, D. L. 1995, AJ, 109, 709

Allen, L. E., Myers, P. C., Di Francesco, J., et al. 2002, ApJ, 566, 993

Allen, L. E., Calvet, N., D'Alessio, P., et al. 2004, ApJS, 154, 363

Baraffe, I., Chabrier, G., Allard, F., \& Hauschildt, P. H. 1998, A\&A, 337, 403

Baraffe, I., Chabrier, G., Barman, T. S., Allard, F., \& Hauschildt, P. H. 2003, A\&A, 402, 701

Barsony, M., Kenyon, S. J., Lada, E. A., \& Teuben, P. J. 1998, VizieR Online Data Catalog, 211, 20109

Barsony, M., Ressler, M. E., \& Marsh, K. A. 2005, ApJ, 630, 381

Bondar', N. I. 2002, Astron. Rep., 46, 489

Bontemps, S., André, P., Kaas, A. A., et al. 2001, A\&A, 372, 173

Bouvier, J., \& Bertout, C. 1989, A\&A, 211, 99

Bouvier, J., Cabrit, S., Fernandez, M., Martin, E. L., \& Matthews, J. M. 1993, A\&A, 272, 176

Buchanan, C. L., Gallimore, J. F., O’Dea, C. P., et al. 2006, AJ, 132, 401

Caballero, J. A., Béjar, V. J. S., Rebolo, R., \& Zapatero Osorio, M. R. 2004, A\&A, 424, 857

Calvet, N., \& Hartmann, L. 1992, ApJ, 386, 239

Carpenter, J. M., Hillenbrand, L. A., \& Skrutskie, M. F. 2001, AJ, 121, 3160

Carpenter, J. M., Hillenbrand, L. A., Skrutskie, M. F., \& Meyer, M. R. 2002, AJ, 124,1001 
Casali, M., Adamson, A., Alves de Oliveira, C., et al. 2007, A\&A, 467, 777 Chabrier, G., Baraffe, I., Allard, F., \& Hauschildt, P. 2000, ApJ, 542, 464 Comeron, F., Rieke, G. H., Burrows, A., \& Rieke, M. J. 1993, ApJ, 416, 185 Dye, S., Warren, S. J., Hambly, N. C., et al. 2006, MNRAS, 372, 1227 Eiroa, C., Oudmaijer, R. D., Davies, J. K., et al. 2002, A\&A, 384, 1038 Elias, J. H. 1978, ApJ, 224, 453

Enya, K., Yoshii, Y., Kobayashi, Y., et al. 2002, ApJS, 141, 31 Evans, II, N. J., Allen, L. E., Blake, G. A., et al. 2003, PASP, 115, 965 Evans, II, N. J., Allen, L. E., Blake, G. A., et al. 2005, Third Delivery of Data from the c2d Legacy Project: IRAC and MIPS (Pasadena, SSC) Fazio, G. G., Hora, J. L., Allen, L. E., et al. 2004, ApJS, 154, 10

Flaherty, K. M., Pipher, J. L., Megeath, S. T., et al. 2007, ApJ, 663, 1069

Gagné, M., Skinner, S. L., \& Daniel, K. J. 2004, ApJ, 613, 393

Grankin, K. N., Melnikov, S. Y., Bouvier, J., Herbst, W., \& Shevchenko, V. S. 2007, A\&A, 461, 183

Grankin, K. N., Bouvier, J., Herbst, W., \& Melnikov, S. Y. 2008, A\&A, 479, 827 Greene, T. P., \& Meyer, M. R. 1995, ApJ, 450, 233

Hambly, N. C., Collins, R. S., Cross, N. J. G., et al. 2008, MNRAS, accepted Hartmann, L., Megeath, S. T., Allen, L., et al. 2005, ApJ, 629, 881

Harvey, P., Merín, B., Huard, T. L., et al. 2007, ApJ, 663, 1149

Herbig, G. H. 2008, AJ, 135, 637

Herbst, W., Herbst, D. K., Grossman, E. J., \& Weinstein, D. 1994, AJ, 108, 1906

Herbst, W., Bailer-Jones, C. A. L., Mundt, R., Meisenheimer, K., \& Wackermann, R. 2002, A\&A, 396, 513

Hewett, P. C., Warren, S. J., Leggett, S. K., \& Hodgkin, S. T. 2006, MNRAS, 367,454

Hillenbrand, L. A. 1997, AJ, 113, 1733

Imanishi, K., Koyama, K., \& Tsuboi, Y. 2001, ApJ, 557, 747

Johns-Krull, C. M., \& Valenti, J. A. 1996, ApJ, 459, L95

Joy, A. H. 1945, ApJ, 102, 168

Kaas, A. A. 1999, AJ, 118, 558

Kenyon, S. J., Hartmann, L., Hewett, R., et al. 1994, AJ, 107, 2153
Kenyon, S. J., Lada, E. A., \& Barsony, M. 1998, AJ, 115, 252

Lada, C. J., \& Wilking, B. A. 1984, ApJ, 287, 610

Lawrence, A., Warren, S. J., Almaini, O., et al. 2007, MNRAS, 379, 1599

Liseau, R., Lorenzetti, D., Molinari, S., et al. 1995, A\&A, 300, 493

Lombardi, M., Lada, C. J., \& Alves, J. 2008, A\&A, 480, 785

Luhman, K. L., \& Rieke, G. H. 1999, ApJ, 525, 440

Luhman, K. L., Whitney, B. A., Meade, M. R., et al. 2006, ApJ, 647, 1180

Martin, E. L., Montmerle, T., Gregorio-Hetem, J., \& Casanova, S. 1998, MNRAS, 300, 733

Megeath, S. T., Allen, L. E., Gutermuth, R. A., et al. 2004, ApJS, 154, 367

Natta, A., Testi, L., \& Randich, S. 2006, A\&A, 452, 245

Ozawa, H., Grosso, N., \& Montmerle, T. 2005, A\&A, 429, 963

Peacock, J. A. 1983, MNRAS, 202, 615

Porras, A., Jørgensen, J. K., Allen, L. E., et al. 2007, ApJ, 656, 493

Reach, W. T., Megeath, S. T., Cohen, M., et al. 2005, PASP, 117, 978

Reipurth, B., Aspin, C., Beck, T., et al. 2007, AJ, 133, 1000

Rieke, G. H., \& Lebofsky, M. J. 1985, ApJ, 288, 618

Scholz, A., \& Eislöffel, J. 2005, A\&A, 429, 1007

Schultheis, M., Ganesh, S., Glass, I. S., et al. 2000, A\&A, 362, 215

Skrutskie, M. F., Meyer, M. R., Whalen, D., \& Hamilton, C. 1996, AJ, 112, 2168

Treister, E., Urry, C. M., Van Duyne, J., et al. 2006, ApJ, 640, 603

Vrba, F. J., Strom, K. M., Strom, S. E., \& Grasdalen, G. L. 1975, ApJ, 197, 77

Vrba, F. J., Rydgren, A. E., Chugainov, P. F., Shakovskaia, N. I., \& Zak, D. S. 1986, ApJ, 306, 199

Wainscoat, R. J., Cohen, M., Volk, K., Walker, H. J., \& Schwartz, D. E. 1992, ApJS, 83, 111

Warren, S. J., Hambly, N. C., Dye, S., et al. 2007, MNRAS, 375, 213

Welch, D. L., \& Stetson, P. B. 1993, AJ, 105, 1813

Wilking, B. A., \& Lada, C. J. 1983, ApJ, 274, 698

Wilking, B. A., Lada, C. J., \& Young, E. T. 1989, ApJ, 340, 823

Wilking, B. A., Meyer, M. R., Robinson, J. G., \& Greene, T. P. 2005, AJ, 130, 1733 